\title{
Stage 4 neuroblastoma: sequential hemi-body irradiation or high- dose chemotherapy plus autologous haemopoietic stem cell transplantation to consolidate primary treatment
}

\author{
R Luksch*, , M Podda', L Gandola², D Polastri', L Piva ${ }^{3}, \mathbf{R}^{2}$ Castellani ${ }^{4}$, P Collini $^{5}$, M Massimino', G Cefalo', \\ M Terenziani', A Ferrari', M Casanova', F Spreafico', C Meazza', F Bozzi', A Marchianò ${ }^{6}$, F Ravagnani ${ }^{7}$ and \\ F Fossati-Bellani \\ 'Unità di Pediatria, Istituto Nazionale Tumori di Milano, Via Venezian, I-20133 Milan, Italy; ${ }^{2}$ Unità di Radioterapia, Istituto Nazionale Tumori di Milano, \\ Via Venezian, I-20133 Milan, Italy; ${ }^{3}$ Unità di Chirurgia Urologica, Istituto Nazionale Tumori di Milano, Via Venezian, I-20I 33 Milan, Italy; ${ }^{4}$ Unità di \\ Medicina Nucleare, Istituto Nazionale Tumori di Milano, Via Venezian, I-20133 Milan, Italy; ${ }^{5}$ Servizio di Anatomia Patologica e Istologia, Istituto \\ Nazionale Tumori di Milano, Via Venezian, I-20I33 Milan, Italy; 'Servizio di Radiodiagnostica, Istituto Nazionale Tumori di Milano, Via Venezian, \\ I-20133 Milan, Italy; ${ }^{7}$ Senvizio Immunotrasfusionale. Istituto Nazionale Tumori di Milano, Via Venezian, I-20I 33 Milan, Italy
}

The aim of the present study was to evaluate the effectiveness of two consecutive nonrandomised treatment programs applied between 1989 and 1999 at the Istituto Nazionale Tumori of Milan in an unselected cohort of 59 children over the age of one with stage 4 neuroblastoma. Both treatment programs consisted of two phases, the induction of the remission phase and the consolidation phase. The induction of the remission phase consisted of intensive chemotherapy, and remained the same throughout the study period. The consolidation phase consisted of sequential hemi-body irradiation (HBI) (I0 Gy per session, 6 weeks apart) in the first period (1988-June 1994) and sequential high-dose cyclophosphamide, etoposide, mitoxantrone + L-PAM and autologous haemopoietic stem cell transplantation in the second (July 1994-1999). Intention-to-treat analysis revealed a significantly better outcome for patients treated with the second program, the 5-year event-free survival probability being 0.12 for program I and $0.3 \mathrm{I}$ for program $2(P=0.03)$. This finding led us to conclude that sequential $\mathrm{HBI}$ is useless as consolidation treatment. The high-dose chemotherapy adopted in the second program enabled a proportion of patients to obtain long-term survival but, since the clinical results remain unsatisfactory, new treatment strategies are warranted.

British Journal of Cancer (2005) 92, 1984-1988. doi:10.1038/sj.bjc.66026I5 www.bjcancer.com

Published online 17 May 2005

(c) 2005 Cancer Research UK

Keywords: neuroblastoma; sequential hemi-body irradiation; autologous stem cell transplantation

In the past two decades, the prognosis for patients with stage 4 neuroblastoma over the age of 1 year has progressively changed, with a weak but significant improvement in clinical results (Goldsby and Matthay, 2004). This slight improvement can be related to the greater and greater aggressiveness of the treatment: intensified chemotherapy increases the percentage of clinical remissions, the introduction of megatherapy helps to eradicate resistant clones, and the addition of 'maintenance' therapy is expected to control any minimal residual disease (Cheung et al, 1998; Pession et al, 1998; Garaventa et al, 1999; Matthay et al, 1999; Pinkerton et al, 2000). The use of aggressive surgery and radiotherapy to the primary tumour site may also contribute to reducing the risk of local relapse (Kushner et al, 2001; Haas-Kogan et al, 2003; La Quaglia et al, 2004). Nowadays, the majority of clinical trials on advanced neuroblastoma are organised according

*Correspondence: Dr R Luksch, Unità Operativa Pediatria, Istituto Nazionale per lo Studio e la Cura dei Tumori, Via Venezian, I, 20133 Milan, Italy; E-mail: roberto.luksch@istitutotumori.mi.it

Received 18 January 2005; revised 4 April 2005; accepted 5 April 2005; published online 17 May 2005 to the sequence: 'induction' of remission with chemotherapy and local treatment at the primary tumour site, 'consolidation' with megatherapy, and 'maintenance' therapy (Matthay et al, 1999; Berthold and Hero, 2000; De Bernardi et al, 2003). The number of studies focusing on the impact of each treatment modality within this arrangement is still limited, however, and the studies on this issue with a randomised design are rarer still (Ladenstein et al, 1994; Pearson et al, 1994; Matthay et al, 1999; Berthold and Hero, 2000; Pritchard et al, 2005).

In the present study, we describe a mono-institutional experience of two consecutive nonrandomised treatment programs comprising an identical induction phase, followed by two different strategies for the consolidation phase, that is, sequential fractionated hemi-body irradiation (HBI) in the first case and sequential high-dose chemotherapy plus autologous haemopoietic stem cell transplantation in the second. Our aim was to explore the impact of these two strategies on outcome.

\section{PATIENTS AND METHODS}

All children over the age of 1 year with previously untreated stage 4 neuroblastoma diagnosed from 1989 to 1999 at the Istituto 
Table I Treatment programs for stage 4 neuroblastoma over the age of I year, 1989-1999

Consolidation phase

Induction phase

Program-I (January 1989-June 1994)

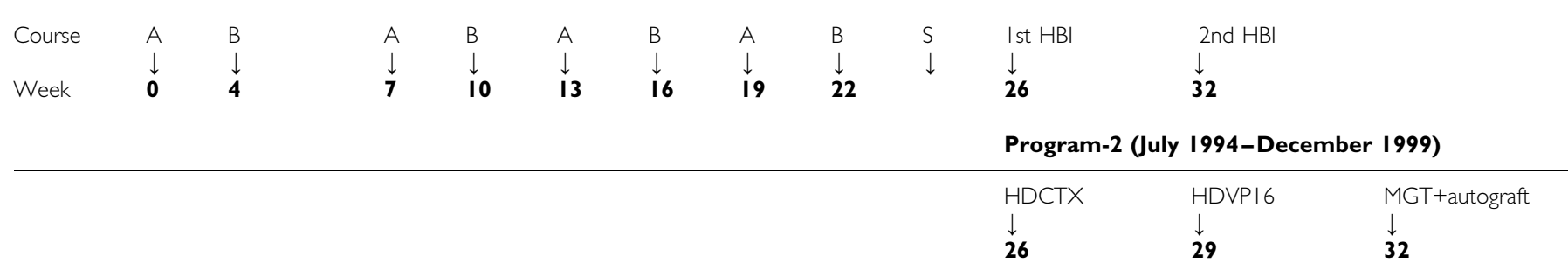

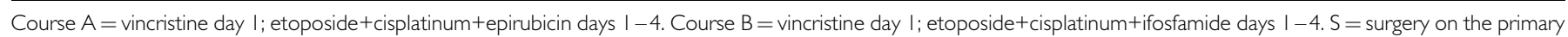

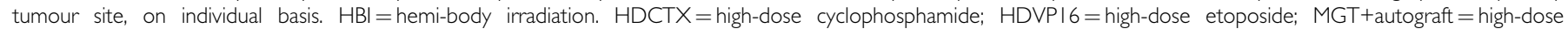
mitoxantrone (day -5$)+$ melphalan (day -2$)+$ autologous stem cell transplantation (day 0 ).

Nazionale Tumori of Milan were prospectively enrolled in the institutional treatment programs.

Diagnosis, staging and response to therapy were evaluated according to the International Neuroblastoma Staging System and the International Neuroblastoma Response Criteria (Brodeur et al, 1993). The diagnosis was based on a histological examination or, in some cases, on the documentation of an unequivocal bone marrow infiltration. The initial evaluation included CT or MRI of the primary tumour, 131-I-mIBG scan, TC-99-MDP scan, bilateral bone marrow biopsy and aspirate, serum levels of LDH, neuronspecific enolase and ferritin, and urinary concentrations of vanylmandelic and homovanillic acids. Response to treatment was assessed after each of the two steps in the treatment strategy (induction and consolidation).

During the study period (1989-1999), two different, nonrandomised treatment programs were adopted (Table 1), both approved by the local Ethical and Scientific Board. The intention was to treat every new child over the age of one admitted to our institution with previously untreated stage 4 neuroblastoma. A stopping rule for toxic deaths was set for both treatment programs. The first program (program-1), applied between January 1989 and June 1994, consisted of an 'induction of remission' phase with intensive chemotherapy and a 'consolidation of remission' phase with sequential fractionated HBI. Briefly, the induction phase consisted of eight cycles of vincristine + cisplatinum + etoposide + epi-adriamycin alternated at 4-week intervals with vincristine + cisplatinum + etoposide + ifosfamide. In each patient, the doses of all drugs except vincristine had to be scaled up by $20 \%$ at each subsequent cycle if the nadir after the previous cycle coincided with WBC $\geqslant 1000 \mathrm{cumm}^{-1}$ and/or platelets $\geqslant 70000$ cumm $^{-1}$. The doses had to be scaled down by $20 \%$ if the nadir coincided with WBC $<1000$ and/or platelets $<70000$ cumm $^{-1}$; the minimal doses given were $100 \%$. At 3 weeks after ending the induction, patients with an objective response (complete, very good, or partial remission) began the consolidation phase. In program-1, this consisted of two consecutive sessions of HBI. The upper and lower body halves were irradiated, with a 6-week interval between the two sessions, using a fractionated technique described elsewhere (Lombardi et al, 1989): $10 \mathrm{~Gy}$, as midplane dose, were delivered in five daily fractions, $2 \mathrm{Gyday}^{-1}$, through two lateral opposite portals at a dosage of $0.2 \mathrm{~Gy} \mathrm{~min}^{-1}$ by a $15 \mathrm{MeV}$ linear accelerator. Program-1 had two aims, that is, to reach an objective response rate higher than the $60 \%$ achieved by the previous treatment program (Lombardi et al, 1989), and to evaluate the impact of HBI on clinical outcome.

The second program (program-2) was adopted between July 1994 and December 1999. This program only differed from the previous one as regards the consolidation phase, which consisted of a sequence at 3-week intervals of cyclophosphamide $7 \mathrm{~g} \mathrm{sqm}^{-1}$, etoposide $2 \mathrm{~g} \mathrm{sqm}^{-1}$, mitoxantrone $60 \mathrm{mg} \mathrm{sqm}^{-1}+$ melphalan $210 \mathrm{mg} \mathrm{sqm}^{-1}$, followed by autologous peripheral blood stem cell transplantation (Table 1). Stem cells were collected from peripheral blood using G-CSF $10 \mu \mathrm{g} \mathrm{kg}^{-1} \mathrm{day}^{-1}$ as of day 4 after administering cyclophosphamide up until the day of leukapheresis. If this target was not reached after cyclophosphamide (at least $3 \times 10 \mathrm{e} 6 \mathrm{CD} 34+$ cells kg $^{-1}$ ), an additional leukapheresis was performed after etoposide, using G-CSF at the same dose as after cyclophosphamide. The aim of program-2 was to evaluate the impact of this consolidation phase on clinical outcome.

In both programs 1 and 2, surgical resection of the primary tumour was on an individual basis and considered as part of the induction of remission phase. In addition, 'local' treatment with radiotherapy was not specified by the treatment plan, and no further treatment was planned after the end of the consolidation phase. Supportive care policies did not change substantially during the study period, with the exception of the use of G-CSF, from 1996 onwards, in cases of febrile neutropenia and documented infection.

In the present study, we describe our mono-institutional experience with the two programs described above. The primary goal of the present study was to assess event-free survival (EFS) and survival (S) probabilities for the two groups of patients enrolled on programs 1 and 2 using an intention-to-treat analysis. Event-free survival was calculated from the first day of treatment up until progression or relapse, or death due to toxicity. Survival was calculated from the first day of treatment until death. EFS and $\mathrm{S}$ distributions were estimated using the Kaplan-Meier method, and compared with the log-rank test. To evaluate the homogeneity of the characteristics of the patients enrolled in programs 1 and 2, the frequency distribution of different variables in the two groups was compared using the $\chi^{2}$ test for: sex, age, site of primary tumour, LDH level, serum ferritin, serum NSE, AVM/HVA dosage, cumulative $\%$ of drug dosages delivered during induction $(\geqslant 120 v s<120 \%$ of the initial dose), surgery on the primary tumour site.

\section{RESULTS}

All 59 consecutive children over the age of 1 year with stage 4 neuroblastoma at onset observed at the Istituto Nazionale Tumori of Milan during the study period were enrolled. In total, 25 joined program-1 and 34 joined program-2. In all cases, the diagnosis, treatment and follow-up were completed at the Istituto Nazionale Tumori. 
Table 3 Response to therapy by treatment program

Table 2 Clinical characteristics at onset and treatment given before consolidation treatment

\begin{tabular}{|c|c|c|c|c|}
\hline & \multicolumn{2}{|c|}{ Program-I } & \multicolumn{2}{|c|}{ Program-2 } \\
\hline & Total $=\mathbf{2 5}$ & $\%$ & Total $=34$ & $\%$ \\
\hline \multicolumn{5}{|l|}{ Sex } \\
\hline Male & 14 & 56 & 19 & 56 \\
\hline Female & 11 & 44 & 15 & 44 \\
\hline \multicolumn{5}{|l|}{ Age ranges (months) } \\
\hline $12-24$ & 9 & 36 & 11 & 32 \\
\hline $25-216$ & 16 & 64 & 23 & 68 \\
\hline \multicolumn{5}{|l|}{ Primary tumour site } \\
\hline Retroperitoneum/adrenal gland & 22 & 88 & 27 & 79 \\
\hline Other sites & 3 & 12 & 7 & 21 \\
\hline \multicolumn{5}{|l|}{ Bone marrow infiltration } \\
\hline Absent & 6 & 24 & 8 & 23 \\
\hline Present & 19 & 76 & 23 & 77 \\
\hline \multicolumn{5}{|l|}{ Bone metastases } \\
\hline Absent & 5 & 20 & 4 & 12 \\
\hline Present & 20 & 80 & 30 & 88 \\
\hline \multicolumn{5}{|l|}{ LDH (56 evaluated) } \\
\hline$<2 n$ & 9 & 37 & 12 & 37 \\
\hline$\geqslant 2 n$ & 15 & 63 & 20 & 63 \\
\hline \multicolumn{5}{|l|}{ NSE (54 evaluated) } \\
\hline$<100 \mathrm{ngml}^{-1}$ & 10 & 43 & 12 & 39 \\
\hline$\geqslant 100 \mathrm{ng} \mathrm{ml}^{-1}$ & 13 & 57 & 19 & 61 \\
\hline \multicolumn{5}{|l|}{ Ferritin (47 evaluated) } \\
\hline$<143 \mathrm{ng} \mathrm{ml}^{-1}$ & 5 & 25 & 9 & 33 \\
\hline$\geqslant 143 \mathrm{ng} \mathrm{ml}^{-1}$ & 15 & 75 & 18 & 67 \\
\hline \multicolumn{5}{|l|}{ VMA/HVA ratio (44 evaluated) } \\
\hline$<1$ & 10 & 62 & 18 & 64 \\
\hline$\geqslant 1$ & 6 & 38 & 10 & 36 \\
\hline \multicolumn{5}{|c|}{ Mean dose of chemotherapy during induction phase } \\
\hline$<120 \%$ & || & 58 & 19 & 70 \\
\hline$\geqslant 120 \%$ & 8 & 42 & 9 & 30 \\
\hline \multicolumn{5}{|l|}{ Surgery on primary tumour site } \\
\hline No & 17 & 84 & 19 & 71 \\
\hline Yes & 2 & 16 & 9 & 29 \\
\hline
\end{tabular}

Abbreviations: $\mathrm{LDH}=$ lactate dehydrogenase; NSE = neuron-specific enolase: VMA = vanylmandelic acid; HVA = homovanillic acid. 47 patients who concluded the induction phase. The distribution of clinical variables at onset, the drug doses given, the number of patients who underwent surgery on the primary tumour site were similar in the two groups (P: NS for all variables).

The patients' demographics and clinical characteristics are shown in Table 2 . The $\mathrm{M} / \mathrm{F}$ ratio was 1.3 , and the median age at diagnosis was 3 years (range 1-18). The site of primary tumour was the retroperitoneum/adrenal gland in the majority of patients $(85 \%)$. Skeleton and bone marrow were the most frequent sites of metastases ( 85 and $72 \%$, respectively), followed by distant lymph nodes $(37 \%)$, liver $(14 \%)$, orbitae $(14 \%)$, central nervous system $(8 \%)$ and lungs $(3 \%)$. The distribution of clinical variables at onset, the drug doses given, the number of patients who underwent surgery on the primary tumour site (Table 2), and the response to induction treatment (Table 3) were similar in the two groups.

All patients received at least two cycles of induction therapy and were evaluable for response. The maximal response during

\begin{tabular}{|c|c|c|c|c|}
\hline & \multicolumn{2}{|l|}{ Program-I } & \multicolumn{2}{|l|}{ Program-2 } \\
\hline & No. of patients & $\%$ & No. of patients & $\%$ \\
\hline \multicolumn{5}{|l|}{ After induction phase (59 patients) } \\
\hline Complete remission & 4 & 16 & 6 & 18 \\
\hline Very good partial remission & 5 & 20 & 5 & 15 \\
\hline Partial remission & 11 & 44 & 16 & 47 \\
\hline No response/progression & 5 & 20 & 7 & \\
\hline \multicolumn{5}{|c|}{ After consolidation phase (47 patients) } \\
\hline Complete remission & 4 & 20 & 16 & 59 \\
\hline Very good partial remission & 7 & 35 & 8 & 30 \\
\hline Partial remission & 8 & 40 & 2 & \\
\hline No response/progression & I & 5 & I & \\
\hline
\end{tabular}

induction was as follows: complete remission, 10; very good partial remission, 10; partial remission, 33; no response (stable disease or progression of disease) 6 . In all, the percentage of responders was $89 \%$. Six of the patients with partial remission experienced progression of disease during the induction treatment, however, so 47 patients concluded the induction phase and entered the consolidation phase. Two additional patients - one after the first HBI session in program-1, and one after high-dose VP16 in program-2 - had progression of disease during the consolidation phase. Thus, 45 patients completed the entire treatment plan, 18 out of $25(72 \%)$ in program-1 and 27 out of $34(79 \%)$ in program-2. Consolidation treatment determined a further response in two out of $19(11 \%)$ after HBI in program-1 and in 16 out of $27(59 \%)$ after high-dose therapy in program-2 (Table 3 ). As for the timing of the treatments, $62 \%$ of cases concluded the treatment program as scheduled, or with a delay $<15$ days, while the remaining $38 \%$ of cases had an overall delay $\geqslant 15$ days (range 15-45). The percentage of cases with a delay of the timing of the treatment in program-1 and program-2 were superimposable (data not shown). For both programs, acute toxicity was mainly haematological. There were no toxic deaths or treatment interruptions due to severe adverse events.

Among the patients who concluded the treatment program, 31 relapsed a median 16 months after diagnosis (range 9-66). The relapse pattern was: metastatic spread, $21(68 \%)$; metastatic spread plus local relapse, eight (26\%); isolated local relapse, two (6\%). None of the patients with nonresponse or progression of disease survived after second-line therapy. In all, 46 out of 59 patients died ( 22 in program-1 and 24 in program-2). The median follow-up for the entire series at the time of the current analysis (as at June 2004) was 62 months (range 51-164). The 5-year EFS and 5-year S probability for the entire series was 0.18 and 0.25 , respectively (Figure 1). Analysing the outcome according to the treatment program adopted revealed a significant difference between the two: the 5-year EFS probability was 0.12 for program-1 and 0.31 for program-2 $(P=0.03)$; the 5-year $S$ probability was 0.12 for program-1 and 0.35 for program-2 $(P=0.03)$ (Figure 2$)$.

\section{DISCUSSION}

Total-body irradiation (TBI) has been widely used in the treatment of advanced NB, mainly as a consolidation strategy, followed by haemopoietic stem cell transplantation (Ladenstein et al, 1994; Matthay et al, 1999). Alternative methods of TBI had also been applied in NB, consisting of cyclic low-dose TBI (D'Angio and Evans, 1983) or sequential HBI in combination with chemotherapy 


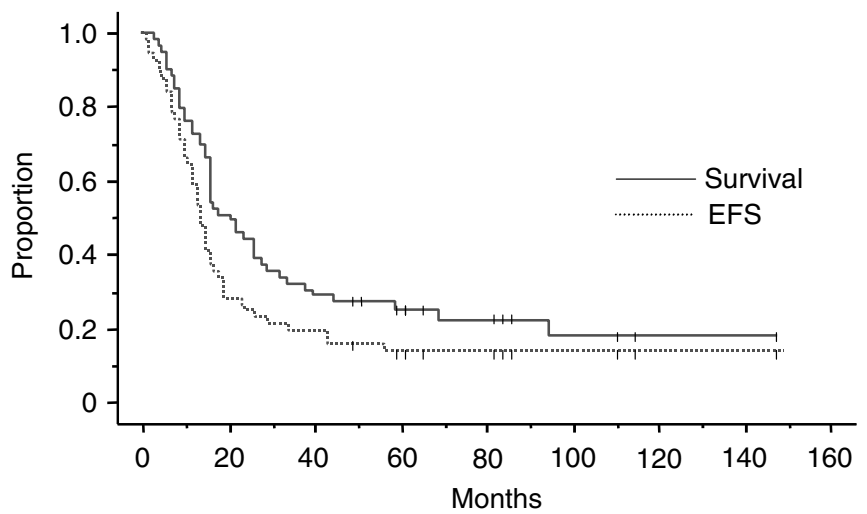

Figure I Event-free survival (EFS) and survival of the entire series.

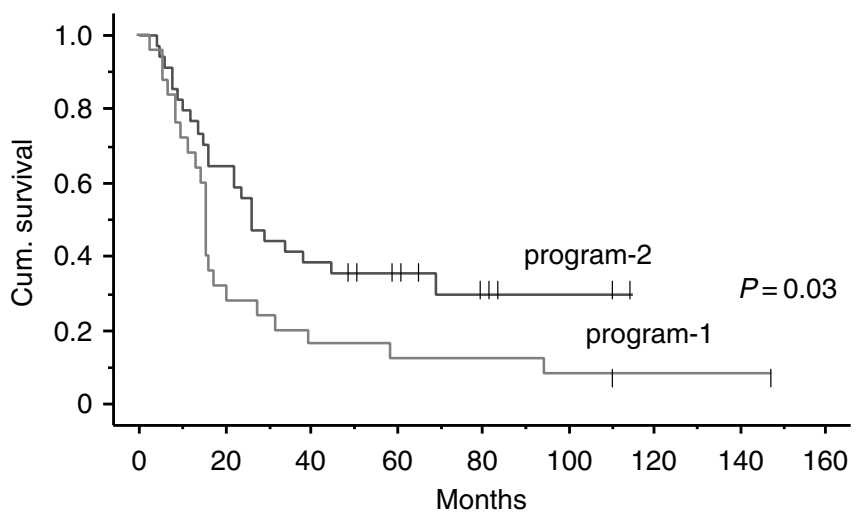

Figure 2 Survival by treatment program.

(Helson et al, 1981). The results of treatment with cyclic irradiation or HBI and concomitant chemotherapy were similar to those obtainable by chemotherapy alone, and were complicated by a more severe toxicity, so these modalities were abandoned. Sequential HBI in advanced NB was also used at the Istituto Nazionale Tumori of Milan, but in a different way, that is, HBI was given as a consolidation strategy after the remission phase, and the results of this experience suggested that fractionated HBI for consolidation after chemotherapy might have a favourable effect on outcome (Lombardi et al, 1989). The promising results of this experience led us to design the program-1 described in the present study in 1989, in which the induction phase with a combination of five drugs in rising doses led to a response rate of $89 \%$, a result comparable with those obtained in other trials (Berthold and Hero, 2000; Pinkerton et al, 2000; Goldsby and Matthay, 2004). Despite the use of intensive chemotherapy, no toxic deaths were encountered and none of the patients had to interrupt the treatment due to toxicity. The clinical outcome of patients treated with program-1 remained poor, however, since the majority had recurrent disease within 24 months of diagnosis despite the high percentage of responders in the induction phase. This led us to conclude that sequential HBI was useless as a consolidation treatment in advanced NB.

Program-2 was designed to try to improve on the results obtained by program-1 by modifying the consolidation phase. Our aim was to explore the role of high-dose sequential cyclophosphamide, etoposide and mitoxantrone + melphalan in erasing residual neoplastic clones proving resistant to the induction phase. Using this schedule, different drugs known to be active in NB were used, with the exception of mitoxantrone, whose activity in NB had been demonstrated in vitro (Fulda et al, 1995) but had never been explored in vivo (Kushner et al, 1990; Pinkerton, 1991; Berthold and Hero, 2000). The consolidation treatment adopted in program2 led us to support the activity of megatherapy in advanced NB, on the grounds of two arguments. The first is the further response obtained with high-dose therapy after the induction therapy in a consistent number of cases - notably higher than the response obtained with HBI. The second is the 5-year survival probability of patients treated with program-2, which was 0.35 - significantly better than for patients treated with program-1.

In the present study, only a small number of patients had surgery on the primary tumour site so we cannot draw any conclusions concerning the impact of a 'local' treatment on the survival probability in this series. Since surgery and radiotherapy on the primary tumour site have been shown to contribute to controlling local relapses in stage $4 \mathrm{NB}$ (Kushner et al, 2001; Haas-Kogan et al, 2003; La Quaglia et al, 2004), it may be that adding a 'local' treatment with surgery plus radiotherapy in an extended way to program-2 might further improve the results we obtained.

We are naturally aware that the limited number of patients enrolled over a lengthy study period and the mono-institutional setting could constitute a bias of our study, and that the statistical results should be interpreted with caution as there might be additional unforseen bias due to the nonrandom design of the treatment programs applied. Furthermore, the possibility that the use of G-CSF could have concurred in the better outcome of program-2 cannot be excluded for certainty. In fact, in the two programs no differences were recorded as to the timing of drug administration schedule and no treatment discontinuation due to acute severe toxicity were recorded in either program. This study included an unselected cohort of patients, the results are described as intention-to-treat, and the survival probability is calculated on the strength of a long follow-up. The use of the same induction treatment throughout the study and the comparable clinical characteristics of the two groups of patients strongly suggest the superiority of the therapeutic results of consolidation treatment with sequential high-dose chemotherapy and autologous stem cell transplantation. Our experience can be added to the limited number of other papers comparing different consolidation strategies after a common induction phase, thus making the results of these strategies fully comparable. These studies evaluated megatherapy $v s$ continuing intensive chemotherapy (Matthay et al, 1999), or maintenance chemotherapy (Berthold et al, 1990; Castel et al, 2001), or no treatment (Pritchard et al, 2005). Combined with the other experiences including megatherapy, they reliably support the conviction that this consolidation treatment modality offers an advantage in terms of survival probability (McCowage et al, 1995; Kushner et al, 2001; Frappaz et al, 2002; Kaneko et al, 2002; Kletzel et al, 2002; De Bernardi et al, 2003).

In spite of all the possible bias, the present study supports the claim that patients with stage $4 \mathrm{NB}$ over the age of 1 year with a tumour responding to initial intensive chemotherapy can benefit from high-dose chemotherapy and haemopoietic stem cell rescue. We are aware that this type of study is not the best way forward and that important clinical questions should nowaday be answered by randomised trials conducted on a multicentre basis with international cooperation. Any chances of improving on the clinical results will obviously come also from new insights on the biology of neuroblastoma and the availability of new active molecules.

\section{ACKNOWLEDGEMENTS}

This work was supported in part by Associazione Bianca Garavaglia, Busto Arsizio (VA). 


\section{REFERENCES}

Berthold F, Burdach S, Kremens B, Lampert F, Niethammer D, Riehm H, Ritter J, Treuner J, Utsch S, Zieschang J (1990) The role of chemotherapy in the treatment of children with neuroblastoma stage IV: the GPOH (German Pediatric Oncology Society) experience. Klin Pädiatr 202: 262 269

Berthold F, Hero B (2000) Neuroblastoma. Current drug therapy recommendations as part of the total treatment approach. Drug 59: $1261-1277$

Brodeur GM, Pritchard J, Berthold F, Brodeur GM, Pritchard J, Berthold F, Carlsen NL, Castel V, Castelberry RP, De Bernardi B, Evans AE, Favrot M, Hedborg F, Kaneko M, Kemshead J, Lampert F, Lee REJ, Look AT, Pearson ADJ, Philip T, Roald B, Sawada T, Seeger RC, Tsuchida Y, Voute PA (1993) Revisions of the international criteria for neuroblastoma diagnosis, staging and response to treatment. J Clin Oncol 11: 1466-1477

Castel V, Canete A, Navarro S, Garcia-Miguel P, Melero C, Acha T, Navajas A, Badal MD (2001) Outcome of high-risk neuroblastoma using a dose intensity approach: improvement in initial but not in long-term results. Med Pediatr Oncol 37: 537-542

Cheung NV, Kushner BH, Cheung IY, Kramer K, Canete A, Gerald W, Bonilla MA, Finn R, Yeh SJ, Larson SM (1998) Anti- $\mathrm{G}_{\mathrm{D} 2}$ antibody treatment of minimal residual stage 4 neuroblastoma diagnosed at more than 1 year of age. J Clin Oncol 16: 3053-3060

D'Angio GJ, Evans AE (1983) Cyclic, low-dose total body irradiation for metastatic neuroblastoma. Int J Radiat Oncol Biol Phys 9: 1961-1965

De Bernardi B, Nicolas B, Boni L, Indolfi P, Carli M, Cordero Di Montezemolo L, Donfrancesco A, Pession A, Provenzi M, di Cataldo A, Rizzo A, Tonini GP, Dallorso S, Conte M, Gambini C, Garaventa A, Bonetti F, Zanazzo A, D'Angelo P, Bruzzi P, Italian Co-Operative Group for Neuroblastoma (2003) Disseminated neuroblastoma in children older than one year at diagnosis: comparable results with three consecutive high-dose protocols adopted by the Italian Co-operative Group for Neuroblastoma. J Clin Oncol 8: $1592-1601$

Frappaz D, Perol D, Michon J, Berger C, Coze C, Bernard JL, Zucker JM, Philip T (2002) The LMCE5 unselected cohort of 25 children consecutively diagnosed with untreated stage 4 neuroblastoma over 1 year at diagnosis. $\mathrm{Br}$ J Cancer 87: $1197-1203$

Fulda S, Honer M, Menke-Moellers I, Berthold F (1995) Antiproliferative potential of cytostatic drugs on neuroblastoma cells in vitro. Eur J Cancer 31A: $616-621$

Garaventa A, Bellagamba O, Lo Piccolo MS, Milanaccio C, Lanino E, Bertolazzi L, Villavecchia GP, Cabria M, Scopinaro G, Claudiani F, De Bernardi B (1999) 131-I-metaiodobenzylguanidine (131-I-MIBG) therapy for residual neuroblastoma: a monoinstitutional experience with 43 patients. Br J Cancer 81: $1378-1384$

Goldsby RE, Matthay KK (2004) Neuroblastoma: evolving therapies for a disease with many faces. Paediatr Drugs 6: 107-122

Haas-Kogan DA, Swift PS, Selch M, Haase GM, Seeger RC, Gerbing RB, Stram DO, Matthay KK (2003) Impact of radiotherapy for high-risk neuroblastoma. A Children's Cancer Group study. Int J Radiat Oncol Biol Phys 56: $28-39$

Helson L, Jereb B, Vogel R (1981) Sequential hemi-body irradiation (HBI) in treatment for advanced neuroblastoma: a pilot study. Int J Radiat Oncol Biol Phys 7: 531-534

Kaneko M, Tsuchida Y, Mugishima H, Ohnuma N, Yamamoto K, Kawa K, Iwafuchi M, Sawada T, Suita S (2002) Intensified chemotherapy increases the survival rates in patients with stage 4 neuroblastoma with MYCN amplification. J Pediatr Hematol/Oncol 24: 613-621
Kletzel M, Katzenstein HM, Haut PR, Yu AL, Morgan E, Reynolds M, Geissler G, Marymount MH, Liu D, Kalapurakal JA, Shore RM, Bardo DM, Schmoldt J, Rademaker AW, Cohn SL (2002) Treatment of high-risk neuroblastoma with triple-tandem high-dose therapy and stem-cell rescue: results of the Chicago pilot II study. J Clin Oncol 20: 2284-2292

Kushner BH, O’Reilly RJ, LaQuaglia M, Cheung NK (1990) Dose-intensive use of cyclophosphamide in ablation of neuroblastoma. Cancer 66: $1095-1100$

Kushner BH, Wolden S, LaQuaglia MP, Kramer K, Verbel D, Heller G, Cheung NK (2001) Hyperfractionated low-dose radiotherapy for highrisk neuroblastoma after intensive chemotherapy and surgery. J Clin Oncol 19: $2821-2828$

La Quaglia MP, Kushner BH, Su W, Heller G, Kramer K, Abramson S, Rosen N, Wolden S, Cheung NK (2004) The impact of gross total resection on local control and survival in high-risk neuroblastoma. J Pediatr Surg 39: $412-417$

Ladenstein R, Lasset C, Hartmann O, Klingebiel T, Bouffet E, Gadner H, Paolucci P, Burdach S, Chauvin F, Pinkerton R, Philip T (1994) Comparison of auto versus allografting as consolidation of primary treatments in advanced neuroblastoma over the age of one year at diagnosis: a report from the European Group for Bone Marrow Transplantation. Bone Marrow Transplant 14: 37-46

Lombardi F, Rottoli L, Gianni C, Gandola L, Lattuada A, Fossati-Bellani F, Gasparini M (1989) Advanced neuroblastoma: results of two treatment programs including sequential hemibody irradiation. Int J Radiat Oncol Biol Phys 17: 485-491

Matthay KK, Villablanca JG, Seeger RC, Stram DO, Harris RE, Ramsay NK, Swift P, Shimada H, Black CT, Brodeur GM, Gerbing RB, Reynolds CP (1999) Treatment of high-risk neuroblastoma with intensive chemotherapy, radiotherapy, autologous bone marrow transplantation, and 13 cisretinoic acid. $N$ Engl J Med 341: $1165-1173$

McCowage GB, Vowels MR, Shaw PJ, Lockwood L, Mameghan H (1995) Autologous bone marrow transplantation for advanced neuroblastoma using teniposide, doxorubicin, melphalan, cisplatin, and total-body irradiation. J Clin Oncol 13: 2789-2795

Pearson ADJ, Pinkerton CR, Lewis IJ (1994) European Neuroblastoma Group fifth study (ENSG5): a randomized study of dose intensity in stage 4 neuroblastoma over the age of one. In Progress in Clinical and Biological Research - Advances in Neuroblastoma Research Evans AE, Biedler JL, Brodeur GM, D’Angio GJ, Nakagawara A (eds) pp 385-394. New York: Wiley-Liss

Pession A, Prete A, Locatelli F, Pierinelli S, Pession AL, Maccario R, Magrini E, De Bernardi B, Paolucci P, Paolucci G (1998) Immunotherapy with low-dose recombinant interleukin-2 after high-dose chemotherapy and autologous stem cell transplantation in neuroblastoma. Br J Cancer 78: $528-533$

Pinkerton CR (1991) ENSG 1-randomized study of high-dose melphalan in neuroblastoma. Bone Marrow Transplant 7(Suppl 3): 112-113

Pinkerton CR, Blanc Vincent MP, Bergeron C, Fervers B, Philip T (2000) Induction chemotherapy in metastatic neuroblastoma - does dose influence response? A critical review of published data standards, options and recommendations (SOR) project of the National Federation of French Cancer Centers (FNCLCC). Eur J Cancer 36: 1808-1815

Pritchard J, Cotterill SJ, Germond SM, Imeson J, de Kraker J, Jones DR (2005) High dose melphalan in the treatment of advanced neuroblastoma: results of a randomised trial (ENSG-1) by the European Neuroblastoma Study Group. Pediatr Blood Cancer 44: 348-357 